\title{
Revisiting the limits of language: The odor lexicon of Maniq
}

\author{
Ewelina Wnuk $^{\mathrm{a}, \mathrm{b}, *}$, Asifa Majid ${ }^{\mathrm{a}, \mathrm{c}, \mathrm{d}}$ \\ ${ }^{a}$ Max Planck Institute for Psycholinguistics, P.O. Box 310, 6500 AH Nijmegen, The Netherlands \\ ${ }^{\mathrm{b}}$ International Max Planck Research School for Language Sciences, P.O. Box 310, 6500 AH Nijmegen, The Netherlands \\ ${ }^{\mathrm{c}}$ Centre for Language Studies, Radboud University, P.O. Box 9103, 6500 HD Nijmegen, The Netherlands \\ ${ }^{\mathrm{d}}$ Donders Institute for Brain, Cognition and Behaviour, Radboud University, P.O. Box 9104, 6500 HE Nijmegen, The Netherlands
}

\section{A R T I C L E I N F O}

\section{Article history:}

Received 28 June 2013

Received in revised form 29 November 2013

Accepted 19 December 2013

\section{Keywords:}

Olfaction

Olfactory naming

Perceptual language

Cross-cultural

Maniq

Aslian

\begin{abstract}
A B S T R A C T
It is widely believed that human languages cannot encode odors. While this is true for English, and other related languages, data from some non-Western languages challenge this view. Maniq, a language spoken by a small population of nomadic hunter-gatherers in southern Thailand, is such a language. It has a lexicon of over a dozen terms dedicated to smell. We examined the semantics of these smell terms in 3 experiments (exemplar listing, similarity judgment and off-line rating). The exemplar listing task confirmed that Maniq smell terms have complex meanings encoding smell qualities. Analyses of the similarity data revealed that the odor lexicon is coherently structured by two dimensions. The underlying dimensions are pleasantness and dangerousness, as verified by the off-line rating study. Ethnographic data illustrate that smell terms have detailed semantics tapping into broader cultural constructs. Contrary to the widespread view that languages cannot encode odors, the Maniq data show odor can be a coherent semantic domain, thus shedding new light on the limits of language.
\end{abstract}

(c) 2014 Elsevier B.V. All rights reserved.

\section{Introduction}

For centuries, scholars and scientists have underestimated the sense of smell in humans. Olfaction is often singled out as the least useful perceptual sense, whose role in life is negligible. "Of all the senses it is the one which appears to contribute least to the cognitions of the human mind" (Condillac, 1754/1930, p. xxxi). Darwin (1874) deemed it to be "of extremely slight service" (p. 17), while to Kant (1798/2006) it appeared as "the most dispensable" (p. 50) of the senses. It has also been claimed that olfaction is of "little special value across cultures" (Gardner, 1993, p. 61) and that man "has left the world of smells" (Burton, 1976, p. 109). Cognitive- and neuro-scientists have expressed the belief that smell is insignificant for humans

\footnotetext{
* Corresponding author at: Max Planck Institute for Psycholinguistics, P.O. Box 310, 6500 AH Nijmegen, The Netherlands. Tel.: +31 24 3521562; fax: +31243521213.

E-mail addresses: ewelina.wnuk@mpi.nl (E.Wnuk), asifa.majid@let.ru. $\mathrm{nl}$ (A. Majid).
}

and that it is "extremely rudimentary" (Grinker, 1934, p. 313), vestigial (Pinker, 1997), or as Stanley-Jones (1957) phrased it, the human rhinencephalon is "untenanted" (p. 594).

Hand in hand with these ideas came the popularization of the belief that olfactory language is impoverished. Sperber (1974/1975), a proponent of the cognitive approach to communication known as Relevance Theory, wrote: "In none of the world's languages does there seem to be a classification of smells comparable, for example, to colour classification... There is no semantic field of smells" (pp. 115-116). According to Henning (1916), "olfactory abstraction is impossible" (p. 66), while Kant (1798/ 2006) remarks on a margin of his manuscript: "Smell does not allow itself to be described, but only compared through similarity with another sense" (p. 51). Similarly, Lawless and Cain (1975) have argued that "Odor quality names (e.g., woody, fruity) are almost always derived from the object from which the odor emanates, and accordingly are not quality names per se" (p. 336). Similar views are found today: 
"the vocabulary of olfaction almost invariably ties the odor to its physical source, e.g., orange or coffee or cheese odors. This is distinctly different than, for example, the vocabulary for color, in which blue, yellow, and red can be distinct percepts themselves, separate from whatever object produces those reflected wavelengths." (Wilson \& Stevenson, 2006, p. 7)

Expert smell vocabularies in Western languages largely reflect that. The flavor and fragrance industry overwhelmingly relies on source-descriptors (e.g., Drake \& Civille, 2003; Martínez-Mayorga et al., 2011; Zarzo, 2008). Lehrer (1983, 2009) presents long lists of source-descriptors produced by wine experts, augmented by metaphorical descriptors, utilizing source-domains as diverse as architecture, artifact production, botany, and music (see also Caballero, 2007). One of the leading contemporary wine critics Robert Parker lists terms such as angular, austere, backward, brawny, decadent, dumb, hot, and unctuous in his glossary of wine terms (Storchmann, 2011; Weil, 2007). It is unclear to what extent these descriptions are conventionalized or whether they even carry any communicative utility at all (Quandt, 2007; Weil, 2007).

Against this backdrop, investigations of language and olfaction have focused heavily on odor identification and odor naming. Unfortunately, these two have not always been adequately distinguished from each other and in many accounts they are treated as equivalent (cf. Jönsson \& Olsson, 2012). Olfactory researchers have nevertheless concluded that odor representations have poor access to language (e.g., Herz \& Engen, 1996; Yeshurun \& Sobel, 2010). If odors are truly inaccessible to language then this has implications for general theories of our underlying cognitive architecture (see Levinson \& Majid, in press).

There is abundant evidence that odor naming is difficult (e.g., Cain, 1979; Cain, de Wijk, Lulejian, Schiet, \& See, 1998; Distel \& Hudson, 2001; Lawless \& Engen, 1977), but the preponderance of data is from English and other closely related languages. The possibility that this may not be universally true is rarely entertained. While several linguistic and anthropological works in the last decades have reported the existence of "smell cultures" with languages rich in abstract odor terms (Beer, 2007; Burenhult \& Majid, 2011; Classen, Howes, \& Synnott, 1994; Hombert, 1992; Lee, 2010; Shepard, 1999; Tufvesson, 2011; van Beek, 1992), these descriptions have not yet reached a wide audience and their full import has not been realized. The psychological literature on olfactory language continues to rely strongly on earlier generalizations, which were made primarily on the basis of WEIRD (Western, Educated, Industrialized, Rich, Democratic) communities (Henrich, Heine, \& Norenzayan, 2010). In this article, we present evidence regarding the cultural and linguistic diversity of olfactory language which has significant bearing on theories of the relationship between olfaction and language. We adopt a multidisciplinary approach, where the perspectives of linguistics, psychology and anthropology are brought together. More broadly, this paper is a further step towards the rapprochement of anthropology and the cognitive sciences that has been recently called for (Beller, Bender, \& Medin, 2012; Bender, Hutchins, \& Medin, 2010; Levinson, 2012).
We examine the olfactory language of the Maniq, a group of nomadic hunter-gatherers living in southern Thailand. The Maniq data challenge the view that olfaction is of little value to humans as well as the idea that olfactory lexica are necessarily impoverished and lacking in abstract terms. At the same time, this paper adds to the literature on olfaction of the larger linguistic group of Aslian (belonging to the Austroasiatic family), which is a locus of considerable olfactory elaboration in the cultural and linguistic realm (e.g., Burenhult \& Majid, 2011; Majid \& Burenhult, 2014; Tufvesson, 2011). This evidence, together with earlier reports of languages with rich smell lexica, opens a new perspective on the language of olfaction.

Studying lexical fields is interesting in its own right, especially in the case of olfaction where specific claims have been made regarding paucity of terminology. In addition, understanding how a semantic field is structured can provide insights into the underlying perceptual system, after all the lexical system must map onto perception in order to enable us to talk about the world. We know from research on other lexical domains that perceptually salient discontinuities are often systematically encoded in languages. For example, Shepard and Cooper (1992) tested normally-sighted participants in two sorting tasks, one involving color chips and another color words. They found that in both cases items were sorted in similar ways so that both color chips and color words were structured according to Newton's color circle. Since the two sortings reflected the same organization, this suggests that the linguistic system faithfully reflects the perceptual system. Other examples of such parallelism include terms for body parts (Majid, 2010; Majid \& van Staden, submitted for publication), gaits (Malt et al., 2008; Malt et al., in press), and biological taxa (Berlin, 1992). If olfaction is similarly structured, then odor terms should mirror odor perception.

Currently, we have only a limited understanding of odor perception and, despite numerous attempts to uncover the structure of the underlying system, there is no agreement on the issue. Proposals regarding the dimensionality of odor perceptual space range from 1 to 32 dimensions (e.g., Khan et al., 2007; Koulakov, Kolterman, Enikolopov, \& Rinberg, 2011; Madany Mamlouk, Chee-Ruiter, Hofmann, \& Bower, 2003; Yeshurun \& Sobel, 2010; Zarzo, 2008). In principle, one could analyze verbal descriptors of smells in English and attempt to relate those to odor perception space but there is a fundamental problem: English smell descriptors are overwhelmingly names of sources (cf. Kaeppler \& Mueller, 2013, who found that 84\% of 175 odor descriptors used in odor classification studies were odor source names). Maniq can, therefore, shed new light on the debate regarding the dimensionality of odor space precisely because, unlike English, it has a dedicated smell lexicon.

There are two questions we ask in this paper. How is the smell lexicon structured? And what does that structure tell us about olfactory perception? Assuming olfactory perception is mirrored in language, and odor perception is onedimensional (cf. Yeshurun \& Sobel, 2010), then the Maniq odor lexicon ought to be one-dimensional too. But if odor perception has larger dimensionality, as suggested by other 
studies (e.g., Kaeppler \& Mueller, 2013), then we ought to find that reflected in the Maniq lexicon as well.

In order to investigate our research questions, in the context of a detailed examination of the olfactory domain as experienced by the Maniq, we draw on the following methods: (1) linguistic elicitation; (2) experimentation; (3) ethnographic observation and interview. We begin with a brief introduction to the Maniq people and language in the next section. We then introduce the smell lexicon of the Maniq, and describe the results of an exemplar listing experiment, described in Study 1. This study explores the meaning of individual Maniq smell terms. Study 2 presents the critical experiment that examines the structure of the olfactory lexicon as a whole. Speakers' similarity judgments of smell terms were collected and analyzed with multidimensional scaling analysis (MDS) and factor analysis (FA) to uncover the dimensions of the olfactory lexicon. In order to identify the concepts underlying these dimensions, an offline rating task of smell terms was conducted in Study 3. In this experiment, people were asked to make judgments of parameters of relevance to olfaction. Together these studies suggest that the Maniq odor lexicon is richly elaborated and coherently structured. The final section of the paper contextualizes this odor lexicon in terms of the indigenous ideology of the Maniq. We discuss smell-related beliefs, practices, and taboos, all of which are crucial for a more complete understanding of the meaning and usage of smell terms.

\section{The Maniq and their language}

Maniq [ma'ni?] is spoken by 250-300 people living in scattered groups in the Banthad mountain range of southern Thailand (more specifically, in Trang, Satun and Phatthalung provinces). Maniq people belong to the larger ethnographic cluster of Semang (cf. Benjamin, 1985) with a traditionally nomadic lifestyle and hunter-gatherer mode of subsistence. Despite on-going deforestation and pressure towards sedentism, many Maniq are still nomadic and continue to hunt animals and forage wild plants. Their economy is further supported by small-scale exchange of forest produce and occasional income from tourists. Maniq bands are highly flexible social structures, often splitting up and regrouping in reaction to the current subsistence conditions. They usually number from 5 to 50 members.

Maniq is a largely undescribed language; previously published resources on the language are limited to word lists (e.g. Bishop \& Peterson, 2003; Phaiboon, 2006). Since 2009, it has been under intense investigation by the first author, who is currently working towards a grammatical description of the language (e.g., Wnuk, in preparation; Wnuk \& Burenhult, submitted for publication). Maniq belongs to the Northern Aslian branch of Aslian, which forms part of the Austroasiatic family. It has a rich phonemic inventory and a highly complex derivational morphology. The default constituent order is SVO (subject-verb-object), with frequent argument ellipsis. The language has rich semantics with fine-grained distinctions encoded in monolexemic forms.

Because of the mobile lifestyle of the Maniq, the remoteness of their habitat and the considerable spread of their campsites, work with a large number of participants is not always feasible. The data presented here were collected in the same area of the Satun province during three successive field trips in 2010, 2011 and 2012.

\section{Language of olfaction}

Talk about smell in Maniq is radically different from that in English. Maniq possesses a rich vocabulary dedicated to describing olfactory sensations. Aside from one exception, ${ }^{1}$ these words are not applicable across multiple sensory domains, but are used exclusively with smell. The terms denote an abstract odor quality without making reference to the source of the smell (unlike, for example, fruity). They are not restricted to a single object, or kind of object, but apply to a diverse range of things. Table 1 in Section 3.1.2 provides a list of 15 Maniq smell terms together with common exemplars.

Odor terminology in Maniq is present in everyday conversation. The smell lexicon is not specialist or known to only a limited group of people. Smell talk is not restricted to particular contexts or registers of speech. It is a mundane activity that all members of the community engage in on a daily basis. Smell is an important reference point in a number of areas of life, such as medicinal practices and rituals (see Section 5). The Maniq constantly monitor odors around them and manage smells so that they are surrounded with healthy and safe scents while avoiding those believed to be hazardous.

Maniq smell terms are phenomenon-oriented descriptions, i.e. they take the experienced odor as the grammatical subject. The controlled activity of smelling as well as the uncontrolled experience of perceiving smell, where the experiencer is the grammatical subject, are both expressed by the verb $2 \tilde{\jmath} n$ 'to smell'. The specific Maniq smell terms do not belong to a single word class. They are encoded in stative verbs (first 10 items in Table 1 ) and noun phrases (the remaining 5 items). Stative verbs are a commonly employed word class across the Maniq lexicon to encode perceptual qualities, e.g. color terms: haypt 'to be black', taste terms: kadek 'to be bitter'. Their meaning is best rendered in English by a phrase "to be" + the adjective referring to a particular quality, e.g. lspas 'to be fragrant, as of tubers, bearcat, new shelter, etc.', or a possessive construction, e.g. 'to have a fragrant smell, as of tubers, bearcat, new shelter, etc.'. Maniq smell terms are not easily rendered into English, so glosses such as 'fragrant' must not be interpreted as direct translations.

Smell stative verbs can take verbal affixes, though they usually do not bear morphology (excluding the frozen iterative morpheme $l s$ - in lspas). A few verbs, namely cancs, cayus and cayə, are semantically and phonologically similar but they do not show evidence of a productive derivational relationship.

Smell noun phrases are headed by the noun $\mathrm{mi}$ ' 'smell'. The phrases are lexical chunks. None of the modifiers, with

\footnotetext{
1 The only exception here is the stative verb baỹ $\phi$ which, in addition to referring to smell, refers to color. It is used for a specific kind of white, e.g. of fog or old person's hair. It is unclear whether and how the smell and color meanings are related to one another.
} 
Table 1

Maniq smell terms with their corresponding exemplars. Numbers in the column indicate the total number of different objects elicited. Numbers in brackets following exemplars indicate the number of consultants who produced that exemplar. Unidentified animal and plant species are given in square brackets.

\begin{tabular}{|c|c|c|}
\hline Smell term & Number of objects & Exemplars \\
\hline cayə & 9 & $\begin{array}{l}\text { tubers (Dioscorea spp.) (4), food (1), cooked food (1), cooked meat (1), rice (1), wild pig } \\
\text { (Sus scrofa) (1), cooked wild pig (1), fresh meat (1), white sun (1) }\end{array}$ \\
\hline cajes & 8 & $\begin{array}{l}\text { animal hair (1), hair of dusky leaf monkey (Trachypithecus obscurus) (1), hair of banded } \\
\text { leaf monkey (Presbytis femoralis) (1), hair of pig-tailed macaque (Macaca nemestrina) (1), } \\
\text { burnt hair (1), burnt animal hair (1), roasted animal fat (1), sun (1) }\end{array}$ \\
\hline canus & 9 & $\begin{array}{l}\text { soap (3), washing oneself (2), fruit (Goniothalamus sp.) (1), leaves (1), Uvaria sp. (1), } \\
\text { clothes (1), talcum powder (1), sun (1), medicine to drink (1) }\end{array}$ \\
\hline hamis & 2 & sun (6), air/smoke coming from the sun (2) \\
\hline ha?ît & 10 & $\begin{array}{l}\text { dead animal (3), rotting animal (3), animal (1), plantain squirrel (Callosciurus notatus) (1), } \\
\text { Prevost's squirrel (Callosciurus prevostii) (1), [wac caw 'kind of squirrel'] (1), bats (1), flying } \\
\text { fox (Pteropus cf. vampyrus) (1), tuber (Dioscorea daunea) (1), bamboo tube (1) }\end{array}$ \\
\hline kameh & 6 & $\begin{array}{l}\text { [taluy 'kind of millipede A'] (5), [caywon 'kind of millipede B'] (1), [ka?ə? basin 'kind of } \\
\text { millipede C'] (1), ipoh poison (Antiaris toxicaria) (1), flying fox (Pteropus cf. vampyrus) (1), } \\
\text { forest (1) }\end{array}$ \\
\hline kamloh & 3 & smoke from fire (3), old shelter (1), bathing (1) \\
\hline lspəs & 14 & $\begin{array}{l}\text { tuber (Dioscorea orbiculata) (2), bearcat (Arctictis binturong) (2), tuber (Dioscorea filiformis) } \\
\text { (1), tuber (Dioscorea calcicola) (1), tubers (Dioscorea spp.) (1), new shelter (1), clean and } \\
\text { dry clothes (1), fruit (Ficus chartacea)(1), forest (1), tree (1), animal (1), food (1), medicine } \\
\text { to drink (1), white sun (1) }\end{array}$ \\
\hline palen & 11 & $\begin{array}{l}\text { blood (3), animal blood (1), blood of wild pig (Sus scrofa) (1), blood of pig-tailed macaque } \\
\text { (Macaca nemestrina) (1), blood of long-tailed macaque (Macaca fascicularis) (1), blood of } \\
\text { bearcat (Arctictis binturong) (1), raw meat (1), [po? batew 'fern sp.'] (1), [smkam 'plant sp.'] } \\
\text { (1), searching for food (1), sun (1) }\end{array}$ \\
\hline ра२ริ? & 16 & 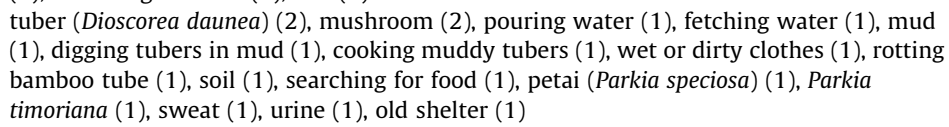 \\
\hline mi? baỹ̃ $\phi$ & 12 & $\begin{array}{l}\text { old shelter ( } 3 \text { ), soil ( } 2 \text { ), shelter ( } 1 \text { ), mushrooms (1), skin of a dead animal ( } 1 \text { ), rotten wood } \\
\text { ( } 1 \text { ), bamboo tube for water ( } 1 \text { ), drinking water from a bamboo tube ( } 1 \text { ), rotten leaf }(1) \text {, } \\
\text { head of banded leaf monkey (Presbytis femoralis) (1), head of pig-tailed macaque (Macaca } \\
\text { nemestrina) (1), head of stump-tailed macaque (Macaca arctoides) (1) }\end{array}$ \\
\hline mi? danəw & 10 & $\begin{array}{l}\text { mushrooms (3), rotten wood (2), rotten mushrooms (1), old shelter (1), animal bones ( } 1 \text { ), } \\
\text { durian seed (1), snakes (1), forest (1), searching for food (1), soil (1) }\end{array}$ \\
\hline mi? huhü $\Phi$ & 10 & $\begin{array}{l}\text { snakes (2), soil (2), searching for tubers (1), digging tubers (1), mushrooms (1), sweat (1), } \\
\text { rotten wood (1), walking in the forest (1), making fire (1), smoke (1) }\end{array}$ \\
\hline mi? latin & 10 & 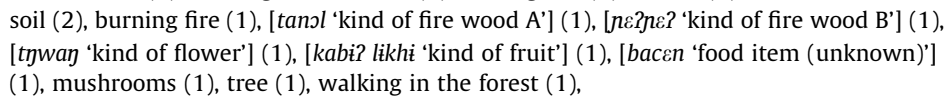 \\
\hline mi? jətu? & 7 & 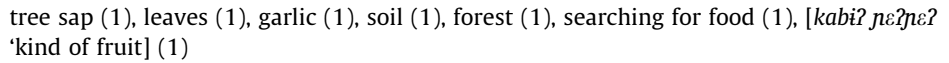 \\
\hline
\end{tabular}

the exception of the stative verb baỹ̃ $\phi$, occur outside the "mi?+..." phrase. For that reason, it is difficult to establish their word class. If they are nouns, it is possible that they originated as source-based terms since ad hoc source-based smell descriptors have the same "mi?+..." structure (e.g. mi? puyo 'smell of rotten trees'; puyo 'rotten tree'). For example, the word danow in mi? danow could have originated from the Malay dangau 'field shelter'. If this is true, the phrase shifted from denoting a smell of one particular object to a more general smell descriptor. This would be similar to what happened to the English color term orange, which first referred to a fruit and only later was used for color (Casson, 1997). However, speakers were not able to provide the meaning of danow (or any of the other modifiers) on their own, which suggests that there is no transparent connection to objects in these terms. In addition, some Maniq speakers derive the modifiers danow, latin and natu? with verbal aspect morphemes, which indicates they might be verbs.

Three items in Table 1 are possible loans from standard Malay and its dialects. Aside from (mir) danıw, the terms are kamsh 'to smell (of millipedes, poison, etc.)' possibly from Borneo Malay kamah 'dirty', and hamis 'to smell (of yellow-colored sun on hot days)' from Malay hamis 'rank in odor, especially of the smell of sweat or an old he-goat, bad belanchan or decaying fish manure'.

\subsection{Study 1: Exemplar listing task}

In order to explore the range of things Maniq smell terms refer to, an exemplar listing task was conducted with speakers. The goal was to uncover what the best exemplars might be for each smell term, and to identify how many different objects participants could readily identify as smelling X.

\subsubsection{Method}

3.1.1.1. Participants. The participants were 8 Maniq speakers ( 4 female) aged approximately $20-50$ years. All were native speakers of Maniq. Six participants contributed responses to the entire (or almost entire) set of smell terms whereas 2 speakers commented on a limited number of terms while another participant was being interviewed. 
3.1.1.2. Stimuli and design. The stimuli were the 15 smell terms in Table 1. The list was compiled on the basis of elicitation sessions and observation of spontaneous language use. Items were presented in different random orders.

3.1.1.3. Procedure. Consultants were verbally presented with the smell terms, one by one, and asked the question "What smells X?", where X was one of the smell terms. Participants were free to list as many exemplars as they wished. The task was carried out in Maniq.

In this first attempt to explore the meaning of smell terms, the task was run in an unconstrained way. Participants were not tested individually and speakers who added responses while another person was being interviewed were not discouraged from doing so. Responses by the same participant given during two or three separate elicitation sessions were also included. In situations where one of the speakers repeated the response heard from another speaker, it was only counted once.

\subsubsection{Results and discussion}

Table 1 lists the terms together with the elicited exemplar sources. Numbers in brackets next to each exemplar indicate the number of consultants who gave that response independently. Participants listed many individual species of plants and animals. The Maniq like many indigenous communities have rich and detailed ethnobiological knowledge (e.g., Medin \& Atran, 1999). Most plants mentioned by the speakers were identified with the help of Maneenoon (2001) and Maneenoon, Sirirugsa, and Sridith (2008). In cases where a plant or animal could not be identified, the Maniq forms are given in square brackets, along with an approximate gloss in English.

Note that exemplar listing is usually done with concrete concepts such as artifacts, natural kinds, and the like (e.g. Ruts et al., 2004). Here we asked people to generate exemplars of abstract concepts, a cognitively more demanding task. This is compounded by the fact that Maniq speakers are non-literate and are not familiar with this sort of task. In spite of this, only occasionally was a participant not able to provide an exemplar. This happened once each with the terms mi? latin, mi? natu?, and kamloh. Overall, people were able to easily generate exemplars, and responses involving 2 or 3 exemplars occurred nearly half of the time. It is striking that smell terms span many different types of objects. Some smell terms have clearly identifiable prototypical sources, e.g. hamis (sun), kameh (millipedes), palky (blood). But even in these cases, people listed additional exemplars. Other terms appear to be more diffuse, e.g. mi? natu? (tree sap, leaves, garlic, soil, forest), mi? baỹ̃ $\phi$ (old shelter, soil, mushrooms, skin of dead animal, etc.). The listed exemplars include edible and non-edible things; plants and animals; single objects, activities as well as locations. This illustrates further the broad applicability of these smell terms and lends credence to the claim that these are abstract olfactory terms.

Interestingly, the listed exemplars for some of the terms are reminiscent of exemplars of smell terms found in the related language Jahai, spoken in Malaysia (Burenhult \& Majid, 2011). When Jahai speakers were asked to generate exemplars for the cognate term of lspas (in Jahai ltpit) they also listed flowers and bearcat (Arctictis binturong) as possible odorants. Similarly for hanit (in Jahai ha? $\tilde{\varepsilon} t$ ) both Maniq speakers and Jahai speakers listed feces and rotten meat. Paley (in Jahai pl?ey) both generated blood and raw meat as exemplars. This suggests a common core to smell terms across these languages.

To summarize, Maniq smell terms are associated with a wide array of exemplars. Smell qualities encoded by the terms are independent of their sources and are applied to different classes of objects.

\section{Organization of the smell lexicon}

Now that we have a better understanding of the individual smell terms, we are in a better position to return to the research questions we posed in the introduction. How is the smell lexicon structured as a whole? And what does that structure tell us about olfactory perception? We assume that part of the meaning of a lexical item is a function of the relations between that term and other items in the domain (e.g., Lyons, 1977; Majid, in press; Saussure, 1967). To get at these meaning relations, we asked participants to perform a similarity judgment task. We separately conducted a rating task in order to further understand the relational structures uncovered. If there is a coherent internal structure to this lexical field then we should be able to model the similarity judgment data produced by the Maniq with a small number of dimensions. The resulting models should have a low stress value while simultaneously accounting for a high percentage of the variance. We test these predictions in the two experiments below.

\subsection{Study 2: Similarity judgment}

We first collected similarity judgments from speakers. Since the Maniq are a non-literate community, we could not conduct a pile-sorting task with words on cards - the usual method for collecting data of this sort (e.g. Shepard \& Cooper, 1992). Instead, we used a triadic comparison task, which does not require reading. People were presented with three smell terms at a time and had to indicate which was the odd one out. The results of the task were then converted into a similarity matrix and analyzed statistically.

\subsubsection{Method}

4.1.1.1. Participants. Eleven Maniq speakers ( 5 female) aged approximately 20-45 years participated in this experiment. All were native speakers of Maniq.

4.1.1.2. Stimuli and design. The stimuli for the experiment were the 15 smell terms in Table 1. A triadic comparison procedure was followed (Weller \& Romney, 1988). A complete triad test with 15 items would result in 455 triads, which is too time-consuming and tiring for participants. So we used a balanced incomplete block design $(\lambda=1)$ of 35 triads instead. The letter $\lambda$ represents "the number of triads in which each pair of items occurs" (Burton \& Nerlove, 1976 , p. 249). We followed the procedures outlined in Burton and Nerlove (1976). Two different triad compositions were created, with each composition presented to half 
of the participants. Items were presented in a pseudo-random order within and across triads to avoid frequent repetition of terms in close proximity.

4.1.1.3. Procedure. Each participant was tested individually in Maniq. Speakers were presented orally with 3 smell terms at a time and asked the following question: "Which one is not the same/similar?" (the meaning of the Maniq term hmin, from Thai my̆an 'same, similar', has scope over both sameness and similarity). The response was coded on a response sheet and the next triad was presented until all triads were complete.

In order to ensure that the task was proceeding as intended, a series of precautions were taken. Before starting the task, the researcher informed the participants that they would be presented with words relating to smell. The critical question was repeated on the initial triads to make sure the participants remembered what they were being asked to do. As they became accustomed to the task, the question was repeated every few triads.

Three objects (three similar leaves from the same plant) were placed in a row in front of the participant to act as anchors to the words in the triad. The researcher would say each smell term while pointing to one of the leaves. The participant could answer by saying the smell term and/or pointing to the corresponding leaf. To prevent participants from falling into a response set, words were assigned to objects sometimes from right to left and other times from left to right. When presenting a triad, target words were pronounced slowly several times with neutral intonation, until the participants made a decision. Many people responded with the following phrases: "These are together" and "This one is alone" or "These are similar" and "This one is not similar". On the rare occasions when a participant could not make a choice after being asked the question several times, the researcher proceeded to the next triad and came back to the problematic case at the end. All participants were able to complete the study.

\subsubsection{Results}

We first tested whether individuals agreed with one another in their similarity judgments of smell words by using cultural consensus analysis (Romney, Weller, \& Batchelder, 1986). Next, we analyzed the data using two independent methods, multidimensional scaling analysis (MDS) and factor analysis (FA), in order to establish the dimensionality of the Maniq smell lexicon.

4.1.2.1. Cultural consensus. In order to establish whether participants agreed in their similarity judgments of the Maniq smell words, we constructed individual $15 \times 15$ similarity matrices for each participant and submitted these to the factor-analytic method described by Romney et al. (1986). If participants agree in their similarity judgments then individuals should load positively on the first extracted factor. The strength of the agreement can be discerned by the size of the eigenvalues (see Boster \& Johnson, 1989). Our solution indicates general consensus for the similarity judgments of smell words. All participants loaded positively on the first factor, and the eigenvalue of the first factor (3.22) was 2.5 times larger than the second (1.33).
We examined loadings of individuals on the second factor for potential sub-group differences between participants, e.g., are people more alike in their similarity judgments based on age, gender, etc. No sub-group patterns emerged. Given the lack of individual differences, we summed all participants in a single aggregate similarity matrix which we then submitted to MDS and FA.

4.1.2.2. Multidimensional scaling analysis. The aggregate matrix with similarities served as input into the multidimensional scaling procedure carried out with the use of the PROXSCAL algorithm in SPSS. To determine the best-fitting solution from MDS, dimensions are added until the stress is sufficiently low and additional dimensions do not result in significant stress reduction. A stress value of 0 would indicate a perfect fit to the data. According to the scree test (Cattell, 1966; Kruskal \& Wish, 1978), number of dimensions can be established by determining where stress values level off. The stress values for solutions from 1 to 6 dimensions were: .188, .098, .055, .054, .045 and .036. The scree test indicates that 2 dimensions are needed to model this data. The stress value of .188 for a one-dimensional solution is poor (Kruskal, 1964), and a pronounced improvement is noted with the addition of a second dimension. A unidimensional solution for this data can therefore be ruled out. A stress value of .098 achieved after adding the second dimension is satisfactory not only from the point of view of the commonly applied scree test, but it is also statistically robust. Sturrock and Rocha (2000) modeled the probability distribution of stress values for over 500,000 random differently sized matrices. According to their calculations, our 2-dimensional solution for 15 items differs significantly from chance $p<.01$. Stress reductions brought by extra dimensions are not substantial, indicating a 2-dimensional representation is optimal for this data set.

In addition, the dispersion-accounted-for (DAF) value for each solution shows an improvement with the addition of the second dimension (from .964 to .99) but only a minor increase with subsequent dimensions (e.g. from .99 of a 2dimensional to .996 of a 3-dimensional solution). Tucker's coefficient of congruence shows the same pattern - for 13 dimensions the values are $.982, .995, .998$. A value of 1 would indicate perfect fit. Taken together with the stress values, this confirms a 2-dimensional solution for this data.

Fig. 1 shows the corresponding MDS plot. Items are more densely concentrated in the left-hand side, while the righthand side is more sparsely populated, with an almost empty area in the upper right quarter.

4.1.2.3. Factor analysis. The same similarity matrix was fed into a factor analysis. The analysis was carried out in SPSS with the principal component extraction method and rotated to a varimax criterion. ${ }^{2}$ Extraction was based on factors with eigenvalues greater than 1 (Kaiser, 1960). This is one of the most commonly used methods to determine the number of factors to extract. Using this criterion four factors were extracted, together accounting for $82.1 \%$ of variance. Using the

\footnotetext{
2 The same number of factors are also extracted without the varimax rotation.
} 


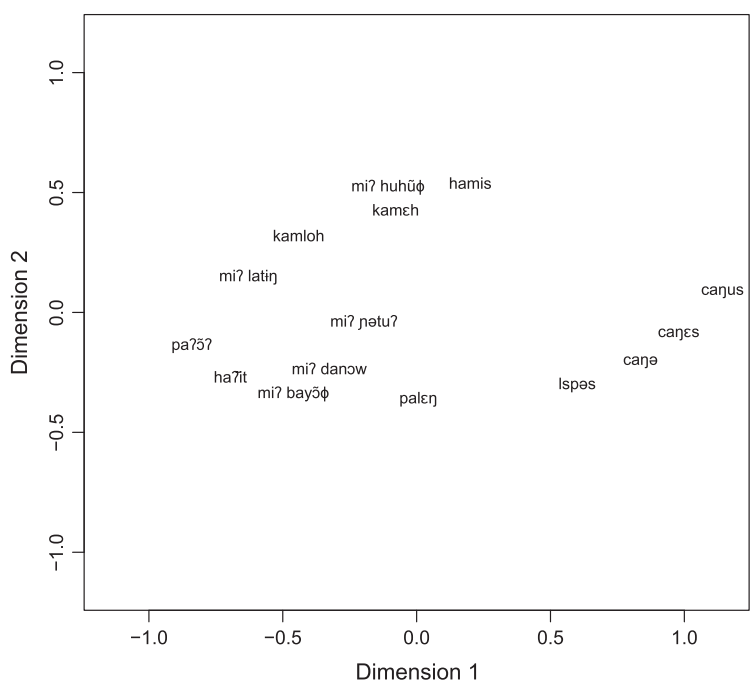

Fig. 1. Two-dimensional MDS based on speakers' similarity judgments of Maniq smell terms.

Table 2

Actual and random eigenvalues according to parallel analysis.

\begin{tabular}{lll}
\hline $\begin{array}{l}\text { Actual } \\
\text { eigenvalue }\end{array}$ & $\begin{array}{l}\text { Average } \\
\text { eigenvalue }\end{array}$ & $\begin{array}{l}\text { 95th percentile } \\
\text { eigenvalue }\end{array}$ \\
\hline 5.844937 & 3.224935 & 3.826036 \\
3.251385 & 2.601913 & 2.99028 \\
1.81112 & 2.119216 & 2.457615 \\
1.413316 & 1.752544 & 1.963543 \\
0.819789 & 1.384843 & 1.592559 \\
0.610867 & 1.126564 & 1.30328 \\
0.426021 & 0.882021 & 1.112897 \\
0.341 & 0.683838 & 0.840399 \\
0.214289 & 0.496494 & 0.661576 \\
0.127082 & 0.333629 & 0.439698 \\
0.097977 & 0.213304 & 0.323811 \\
0.037209 & 0.116883 & 0.195174 \\
0.003767 & 0.05227 & 0.113802 \\
0.001241 & 0.011544 & 0.038899 \\
\hline
\end{tabular}

scree test of Cattell (1966) a plausible case could also be made for two factors to be retained. Therefore, at least two but up to four factors are required to model this data. It has been argued, however, that both of these criteria are subjective. Therefore, parallel analysis (PA) is recommended instead as an objective method to determine number of factors to retain. In PA multiple matrices with the same parameters as the raw data are constructed and the resulting eigenvalues from the randomly permuted matrices are compared to the original dataset (Hayton, Allen, \& Scarpello, 2004). Following O'Connor (2000), we created 100 permuted datasets and conducted FA over these matrices. We then calculated the average eigenvalues and 95th percentiles for the datasets. Comparing these values to those for the original data shows that only the first two factors of the original data had eigenvalues greater than that of the permutated data (see Table 2). Therefore, a two-factor solution is supported.

Using multiple criteria, it is clear that a unidimensional solution is inadequate, and the optimal solution has 2 factors accounting for $60.6 \%$ of variance (factor $1=39 \%$, factor $2=21.7 \%)$.

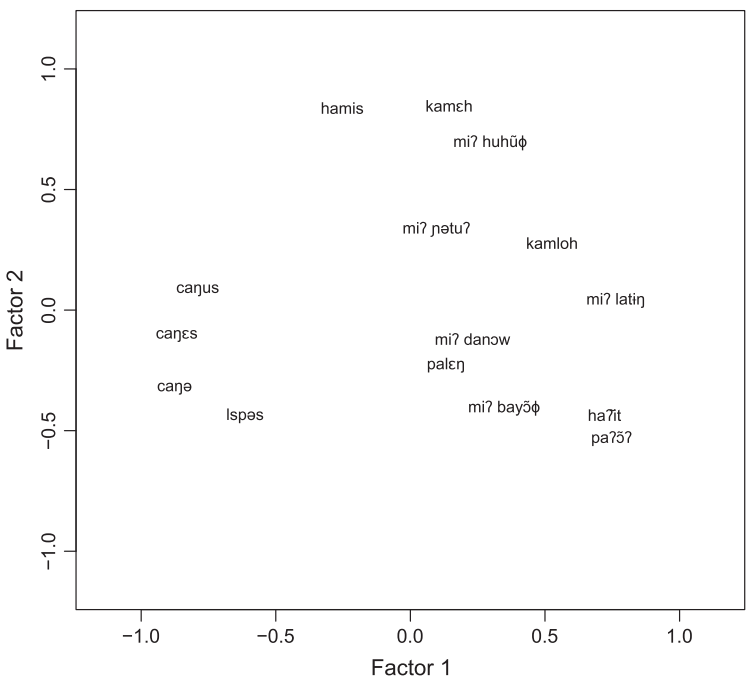

Fig. 2. Two main factors of a varimax-rotated FA based on speakers' similarity judgments of Maniq smell terms.

The FA plot in Fig. 2 echoes the MDS plot in Fig. 1, although items on the first dimension are plotted in mirror-image. There is a similar circular ordering of terms in both analyses.

\subsubsection{Discussion}

We asked in the Introduction: How is the smell lexicon structured in Maniq? Both MDS and FA converge to show that the Maniq smell lexicon is a coherent lexical field with internal structure. One dimension is not sufficient to adequately model the similarity data. Standard tests indicate that the optimal solution is 2-dimensional; however, since $40 \%$ of variance remains unaccounted for in the FA, it is possible that additional factors, not captured by the scaling model, also play a role.

What are the implications of such an outcome for theories of smell perception? The multidimensional structure of the Maniq smell lexicon is incompatible with unidimensional models of odor (Yeshurun \& Sobel, 2010). It also does not support solutions of extremely high dimensionality (e.g. Madany Mamlouk et al., 2003). The perceptual models most closely approximating the structure found for the Maniq olfactory language are 2- to 4-dimensional, with parsimony suggesting a 2-dimensional solution (e.g. Koulakov et al., 2011).

Can the first two dimensions be labeled or are they themselves ineffable? A closer examination of the terms, and their associated meanings revealed in Study 1, suggests that the first dimension might tap into pleasantness. Terms referring to pleasant and unpleasant smells lie at the opposite ends of dimension 1 . The interpretation of the second dimension is less clear. There are at least two plausible interpretations. Either the terms on this dimension refer to smells that are edible vs. inedible or to smells that are dangerous vs. safe. This is based on the observation that terms at the top describe smells of dangerous inedible objects (e.g. snakes, sun) while those at the bottom have among their exemplars edible non-dangerous objects (e.g. 
tubers, petai). In order to test these interpretations, we conducted a rating study. Participants were asked to rate the 15 smell terms on a number of parameters salient for olfaction, and the ratings were then correlated with the MDS and FA solutions.

\subsection{Study 3: Rating task}

As discussed earlier, the main dimension implicated in smell perception is pleasantness (e.g., Khan et al., 2007; Yeshurun \& Sobel, 2010). But other studies have made finer-level distinctions in the parameters that might be relevant for smell perception. For example, Chrea et al. (2004) asked American, French and Vietnamese participants to rate odors according to pleasantness, but also familiarity, intensity, cosmetic use and edibility. In another perceptual judgment task, Rouby and Bensafi (2002) asked participants to evaluate odors according to pleasantness, intensity and dangerousness. These parameters were shown to be relevant for evaluating real odors. We ask here whether similar parameters may be relevant for odor terms.

Maniq speakers were asked to rate odor terms on an 8-point scale for pleasantness, edibility, familiarity, dangerousness, cosmetic value and intensity. Pleasantness, edibility and dangerousness were chosen because we hypothesized earlier that they could explain the dimensions in the MDS and FA plots. Cosmetic value was deemed important because it discriminated between odor clusters in Chrea et al. study (2004). Familiarity and intensity were selected because they are reported to influence odor perception (e.g. Ayabe-Kanamura et al., 1998; Doty, 1975).

\subsubsection{Method}

4.2.1.1. Participants. Eight Maniq speakers ( 4 female) aged approximately $20-50$ years participated in this study. All were native speakers of Maniq.

4.2.1.2. Stimuli and design. The same 15 terms from Table 1 were tested. Participants were asked to rate pleasantness, edibility, familiarity, dangerousness, cosmetic value and intensity for each term on an 8-point rating scale. Items were presented in a fixed random order to all participants. Since Maniq speakers were not familiar with this sort of task, we determined that ratings by numbers or by pointing to a line anchored by numbers would not be appropriate. So we elicited ratings by presenting people with a line of circles of increasing size instead. Participates made judgments by pointing to the smallest circle to indicate the lowest value and the largest circle to indicate the highest value. High ratings were associated with extremely pleasant, edible, familiar, etc., while low ratings were associated with unpleasant, inedible, unfamiliar, etc.

Table 3 gives the English translations of the Maniq phrases used in the task. Most of the scales were easily translated. Cosmetic value was operationalized as suitability to be used in a necklace because the Maniq often wear fragrant necklaces. Intensity was expressed with the size metaphor - strong smells were said to be big, while weak ones small. This was based on the use of a size metaphor to express the notion of intensity in other domains, e.g. in sound.

Rating on each of the scales was preceded by a brief training period. Training items were selected on the basis of structural and semantic resemblance to smell terms (i.e., stative verbs with abstract meanings), yet they were distinct in that they could not be employed as odor descriptors. Examples of the training items include: "bitter", "spotted", "soft", "black" and "heavy".

4.2.1.3. Procedure. Participants were tested individually and the task was run in Maniq. First the rating scale was introduced. The experimenter explained the concept of pleasantness and how it applied to the scale. Previous ethnographic work provided the relevant information to exemplify each of the parameters according to indigenous beliefs.

For pleasantness, the experimenter pointed to the largest point on the scale and gave an example of a saliently pleasant property, "fatty/starchy", adding that it was "really good" and that this point represented "really good". She then indicated the smallest point and gave an example of a saliently unpleasant training item, "bitter", adding that it was "not good" and that this point represented "not good". Finally, the experimenter pointed to the middle circle and gave an example of an item which was neutral, "bland", adding that it was "somewhat good" and that this point represented "somewhat good". After the introduction to the pleasantness scale, the participant was asked to rate the pleasantness of several items from the taste domain (sour, sweet, chalky, spicy, salty). Once the participant understood the task, the experimenter moved to pleasantness judgments of the experimental items. Ratings for other parameters were collected in the same way.

\subsubsection{Results and discussion}

The rating data was used to test which parameters might underlie the 2-dimensional solutions uncovered by the earlier MDS and FA. We calculated the correlation between each of the scales in the rating task, and the MDS and FA loadings of smell terms. Table 4 presents the results.

Table 3

English translations of Maniq phrases used in the study to denote extreme points of the scale.

\begin{tabular}{llll}
\hline Parameter & Smallest point & Mid area & Largest point \\
\hline pleasantness & not good & somewhat good & eat a bit \\
edibility & not eat & know a bit & eat a lot \\
familiarity & not know & fear a bit & know well \\
dangerousness & not fear & use a bit to make necklaces a lot & somewhat big \\
cosmetic value & not use to make necklaces & & big a lot to make necklaces \\
intensity & not big & &
\end{tabular}


Table 4

Correlations between average ratings of smell terms and their loadings on the dimensions of the MDS and FA.

\begin{tabular}{|c|c|c|c|c|c|c|}
\hline & Pleasant & Edible & Familiar & Dangerous & Cosmetic & Intense \\
\hline \multicolumn{7}{|l|}{ MDS } \\
\hline Dimension 1 & $.888^{* * *}$ & $.749^{* *}$ & $.756^{* *}$ & $-.543^{*}$ & $.823^{* *}$ & $.782^{* *}$ \\
\hline Dimension 2 & -.256 & -.350 & -.170 & $.527^{*}$ & -.229 & -.345 \\
\hline \multicolumn{7}{|l|}{$F A$} \\
\hline Factor 1 & $-.908^{* * *}$ & $-.798^{* * *}$ & $-.763^{* *}$ & $.602^{*}$ & $-.854^{* *}$ & $-.813^{* *}$ \\
\hline Factor 2 & -.237 & -.399 & -.269 & $.526^{*}$ & -.254 & -.343 \\
\hline
\end{tabular}

** $p<.01$.

$p<.05$.

Correlations between rating judgments and the loadings on the MDS and FA plots show the same pattern. As hypothesized, the first dimension correlates most highly with pleasantness. It also correlates significantly with all other parameters. This is not surprising since edible, familiar, and cosmetically valued things are usually pleasant, while dangerous things are unpleasant. It is not clear why intensity shows a correlation since this could be argued to be an independently varying factor. This result requires further investigation. It is possible that the size metaphor was not understood as intended despite our precautions. The second dimension correlates significantly only with dangerousness. Note that dangerousness is not just an inverse of pleasantness. The second dimension appears to tap a distinct construct - the arousal and ensuing alertness provoked by smell. To illustrate, the three terms mi? huhü $\phi$, hamis and kamsh appear at the top of dimension 2. They refer to the smells of snakes, sun, millipedes and poison. Because these smells and smell sources are believed to inflict pain, illness and death, the Maniq fear them. These odors and odor terms trigger alertness so the person is ready to respond to the accompanying dangers. The relevant responses can be different for each object. Terms on the other side of the axis, mi? baỹ̃ $\phi$, lspas and ha?it, are not usually associated with fear and all received relatively low ratings on the dangerousness scale. There is, however, an exception. Palı $\eta$ received a high score. This apparent inconsistency can be understood by recognizing the internal complexity of the term, whose meaning combines several distinct notions. On the one hand, palen refers to the smell of blood and raw meat (which are not dangerous for the Maniq) and, as indicated by the exemplar listings, this is the most prototypical aspect of the term. On the other hand, palen refers to the smell of windstorms and softened pandan leaves, both of which are believed to provoke abdominal pain. In the similarity judgment task, participants appear to have focused on the prototypical meaning while in the rating task the "dangerous" aspect likely brought to mind the less prototypical exemplars that fit the dangerous criteria resulting in relatively high scores on the scale.

How do these findings correspond to odor perception studies? Overwhelmingly, previous studies have found that the first dimension in odor perception is best accounted for by pleasantness (e.g. Khan et al., 2007; Koulakov et al., 2011; Zarzo, 2008). Pleasantness is found to correlate with the first PC (principal component) of odor molecular structure (Khan et al., 2007) and the first PC of olfactory neural activity (Haddad et al., 2010). The fact that we find it to be important for olfactory language lends further support to the idea that pleasantness is of primary importance in olfaction. Our second dimension of dangerousness also has support in the odor perception literature. According to Haddad et al. (2010), the second dimension of odor perception reflects toxicity, a notion closely related to dangerousness. The parallelism between the structure of the Maniq odor lexicon and previous odor perception studies conducted independently is highly suggestive of the broader notion that language reflects structure in the world. Future research simultaneously exploring odor perception and odor language in Maniq is required to unpack this further.

To summarize, the Maniq smell lexicon can be characterized by a low-dimensional space, optimally modeled in 2 dimensions, and which correlate with pleasantness and dangerousness. ${ }^{3}$ The identification of a pleasantness and dangerousness dimension is in line with the established view that pleasantness is the primary axis of odor perception (Khan et al., 2007), as well as the more recent proposal that toxicity is a secondary dimension in odor perception (Haddad et al., 2010). Overall, our results lend support to low-dimensional odor perception models such as the 2-dimensional model of Koulakov et al. (2011).

The cognitive structure uncovered by the MDS and FA reveals how the Maniq olfactory lexicon is organized. It does not provide us with a complete semantic analysis of the lexical field, but - by uncovering how the terms relate to one another - it brings us a step closer to understanding the semantics of Maniq odor terms. Before concluding this paper, we would like to turn briefly to the role of odor in Maniq culture in order to further flesh out their semantic richness and illustrate the significance of smell.

\section{The significance of smell in indigenous beliefs and practices}

Maniq smell categories are culturally and linguistically elaborated. Smell is an important part of the indigenous concepts of well-being and safety, and therefore it is intertwined with knowledge from other domains, such as

\footnotetext{
${ }^{3}$ Although there is a tradition of interpreting scaling results of similarity judgments as revealing the semantic structure of a lexical domain, we do not intend to suggest that the current results are a full semantic analysis of odor terms in Maniq (for interpretive pitfalls along these lines, see Fillenbaum \& Rapoport, 1971, 1974). Providing a complete account of the lexical meaning of odor terms would require a deeper understanding of their full extensional ranges, sense relations, and conditions of usage, arrived at by explicit tests as well as observations in context.
} 
ethnobiology, natural phenomena, and the supernatural world. This background is necessary to fully understand the shades of meaning associated with the smell lexical categories.

It is tempting to interpret the smell terms in Maniq as various ways of describing fragrant and foul odors. But the semantics are much more complex, and we posit that the odor terms critically capture specific odor qualities. These meanings are difficult to translate into English since it lacks the relevant terminology. The discussion below illustrates the cultural embeddedness of the meanings conveyed by smell terms.

\subsection{Odor and culture}

According to the Maniq, smell (mi?) is emitted by various organisms and objects in the world. Its prototypical locus is referred to by two expressions: paloh $(y x)$, a loanword from Malay peluh 'sweat', and mina?, its indigenous synonym. The two terms are interchangeable. They both denote the "essence" of an object - a substance (usually liquid) that emits smell. For humans the essence is sweat. For animals, plants and mushrooms, it is an odorous liquid found inside the organism. Paloh is not restricted to living things, however, and is believed to be a property of all kinds of objects that emit odor (e.g. the sun).

Odor does not remain constant over time. There are cases where this is obvious, such as food that changes its smell when processed or left uneaten for too long. There are also cyclic changes that affect which odors are present in the environment. For instance, hog badger (Arctonyx collaris) is described as smelling caya (a good aroma often associated with various foodstuffs) during the dry season. In the wet season, however, the badger has an unpleasant odor similar to a monitor lizard. The smells carry with them implications for hunting. Skinny and bad-smelling badgers are not usually pursued by the Maniq. Fatty ones with an aromatic odor, on the other hand, will cause the group to move camp into the vicinity of the badger's feeding area and hunt it.

Smell is vital in the life of the Maniq. People keep constant track of scents and rely on olfactory information in a variety of situations, from everyday foraging, indigenous medicine to the ritualized use of scents. They also manipulate odors in their immediate environment to counteract possible dangers and maintain a state of balance. A number of objects and natural phenomena associated with danger or disease are said to be accompanied by unpleasant smells. According to the Maniq, violent windstorms are the source of an unpleasant smell referred to by the verb palen. The wind is appeased by neutralizing the bad odor through burning the rhizome of the plant called kasay 'Dianella ensifolia'. This releases a pleasant fragrance, lspas, which helps to counterbalance paley. Paley is also relevant during the preparatory stage of weaving, when pandan leaves are softened. On both occasions (during windstorms and when preparing to weave), paley may provoke abdominal pain, treated by applying metal dust, obtained in the process of sharpening a machete, to the sore spot. Machetes are often used as compresses because of their cool temperature, considered to be one of the two essential elements of a healthy environment (good smell being the other).
Another feared unpleasant smell is hamis, the odor of sun, present in the atmosphere on some hot days when the sun has a yellow color. Those moments are potentially dangerous since both heat and hamis can lead to illness with fever, burnt ("red") eyes and headache. So people seek refuge in their shelters because they believe that a cool and shaded environment is healthy and provides protection against disease. They also perform a ritual where they burn animal hair and bones in order to release a pleasant smell (cayes), which together with the smoke floats up to the sun and eliminates the dangerous hamis (cf. Dallos, 2011; Endicott, 1979).

Just as negative objects and phenomena are accompanied by bad odors, positive ones are often connected to good fragrances. A large number of medicinal herbs collected by the Maniq have intense aromas, the majority of which can be described by the term lspas 'to be fragrant'. Exemplars include: kasay 'Dianella ensifolia', kunit 'turmeric (Curcuma domestica)', biha 'Triomma cf. malaccensis' and $p^{h} l \varepsilon y$ 'Cassumunar ginger (Zingiber montanum)' (Thai phlaj). The fact that pleasing odors and healing or disease-preventive powers come together in a large number of plants speaks to a perceived causal connection. The Maniq believe that medicinal plants worn in necklaces, headbands and wristbands protect them against illness because they are fragrant (lspas). Wearing them is thus a common practice among the Maniq.

A prime example of a fragrant medicinal plant is kasay 'Dianella ensifolia' mentioned before in the context of the wind-appeasing practice. Its rhizome has a number of therapeutic uses and it is administered in a variety of ways. It can be boiled and drunk as an infusion, the smoke produced by burning it in fire can be inhaled, or, if the condition of the patient is very bad, the smoke can be blown over the body by another person. Kasay is used to treat a wide variety of conditions, such as stomachache, muscle/flesh pain and dizziness. When questioned, people do not offer detailed explanations of how smoke counters disease or wind, but a valuable insight into understanding these practices can be gained from the description of the same act performed by the Batek, a closely related group living in Malaysia:

"The smoke is supposed to enter the body and cause the disease to flee. This is because the odour of the smoke is good (bed'èt) and that of the disease bad (jebéc), and they cannot mix. If the smoke goes in, the disease must leave. Alternatively, some say the good-smelling smoke draws the disease out of the body by attracting it, causing it to follow the smoke as it wafts upward from the patient's body." (Endicott, 1979, pp. 107-108)

These beliefs and practices are a vivid illustration of how much power is attributed to odor, belying the claims that odor is of little value across cultures.

\subsection{Returning to smell terms}

The lexical field of smell may be reducible to two dimensions, but the meanings of individual smell terms hook into rich cultural knowledge. Cayus, for example, is the odor of cosmetic products and cleanliness and so it could be thought to be unambiguously pleasant. It lies on the 
extreme end of the pleasant dimension in Figs. 1 and 2. But the fruit kulsw (unidentified) is also cajus, even though it is poisonous. Maniq avoid the fruit. Leaf monkeys, on the other hand, feed on kulsw fruit. Leaf monkeys are also a common food for the Maniq. However, if the Maniq hunt and eat a leaf monkey who has recently consumed the kulsw fruit, they will become sick. A monkey which has eaten kulsw is said to smell canus, just like the fruit - a warning for people that they should not eat it in that state.

Lspos also lies at the extreme end of the pleasant spectrum. This is the smell of food and fragrant (and medicinal) plants. However, lspəs smells must be handled carefully and if not this can lead to dire consequences. According to a taboo referred to as Pamse?, it is forbidden to mix the lspəs smell with game. More specifically, people must not consume lspəs-smelling plants or drinks with the meat of hunted animals. It is also prohibited to touch or wear lspəs plants before or during direct contact with game. The consequences of breaching this taboo depend on the particular fragrant plant involved. If it is cawes (unidentified plant), salih (Alpinia sp.), or hubiew (unidentified plant), it will bring about Pamse? ta?ว? - an attack by a streak of tigers. The tigers will ambush the Maniq campsite and attack people by biting at their heads or eyes. According to some people, the attack could also take place during a hunting trip, suddenly and unexpectedly. The consequences are far less serious if the fragrant plants are biha (Triomma cf. Malaccensis), lawen (Cinnamomum subavenium or Neolitsea sp.), gale (Uvaria sp.), tin dik diew (Goniothalamus sp.) (Thai ching dכok diaw) or caylun (Elettariopsis sp.). Mixing these with game causes Pamse? dok, i.e. reduced effectiveness of dart poison used in hunting. Hunts will be unsuccessful despite accurate shooting. Of the two, Pamse? ta?o? is certainly more salient. In fact, some Maniq consider the combination of all lspəs-smelling plants with game as being equally likely to expose them to a tiger attack.

Although mixing lspəs smells with game is dangerous, there is an odor remedy: the smell of dart poison, kamsh, released by burning toxic plants (e.g. baicl 'Strychnos sp.') or the processed dart poison. Kamch is said to block the tiger's nose, thereby confusing it, so that the tiger cannot find its way to the campsite. Kamch itself is ambiguous as to whether it relates to something good or bad (it lies in the middle of dimension 1 in Figs. 1 and 2). On the one hand, it can be a weapon and protection; on the other hand, it is a poison found in venomous millipedes.

These examples illustrate the complex cultural constructs that odor terms tap into. Distinctions such as good-bad or alert-calm are important in distinguishing terms from one another, but they are not necessarily fixed. The parameters pleasantness and dangerousness are malleable and depend on the contexts considered.

\section{Discussion}

Smell is particularly important in the lives of the Maniq, and lies at the core of the indigenous ideology. The Maniq surround themselves with odors believed to be beneficial for health and that repel danger. They stay constantly alert for potentially harmful odors. Olfactory knowledge is central to the life of the Maniq as it is deeply interconnected with knowledge in other principal areas of daily experience such as ethnobiology and natural phenomena. This shows that claims such as odors have "little special value across cultures" (Gardner, 1993, p. 61) are simply wrong.

Alongside the cultural preoccupation with odors, we have shown the Maniq language possesses a rich odor vocabulary of over a dozen abstract terms. This is evidence against the long-standing and widespread view that humans do not have words for smells, or that the language of odor is non-abstract and steeped in metaphors (e.g., Kant, 1798/2006; Lawless \& Cain, 1975; Wilson \& Stevenson, 2006). Maniq smell terms refer to different types of odor qualities. They apply to a variety of objects, as exemplified in Study 1, and tap into broader cultural constructs.

The Maniq system is remarkable because there are dedicated terms for olfaction and these are part of everyday talk. Western languages simply lack this. When describing smells, English speakers, for example, most often resort to source-descriptors, such as like a banana or fruity, but even these descriptors are not applied accurately (e.g., Cain, 1979; Majid \& Burenhult, 2014). Experts also largely rely on source-descriptors. As the wine writer and columnist Gluck (2003) states:

"We wine writers are the worst qualified of critical experts. This is largely, though not exclusively, because we are the most poorly equipped. The most important tool at our disposal is inadequate for the job. That tool is the English language." (p.107)

We showed in Study 2 that Maniq smell words constitute a coherent semantic field (contra Sperber, 1974/1975, and others). The 2-dimensional structure uncovered in our study argues in favor of low-dimensional odor perceptual spaces (e.g. Koulakov et al., 2011). Study 3 showed further that the first dimension correlated with pleasantness and the second dimension with dangerousness. The fact that most of the variance in the data is explained by pleasantness and dangerousness bolsters the claim that the hedonic dimension plays a primary role in smell perception (cf. Khan et al., 2007; Koulakov et al., 2011; Zarzo, 2008) and provides support for toxicity as the second dimension of smell perception (Haddad et al., 2010).

The 2-dimensional structure we uncovered is reminiscent of the similarity space of emotion terms discovered by Russell (1980; Russell, Lewicka, \& Niit, 1989). The circumplex model of emotion has been characterized by a pleasure-displeasure dimension and a degree-of-arousal dimension. We have argued that these constructs are plausible interpretations of our 2-dimensional solution of odor terms in Maniq too. Why might we find these two dimensions for odors? It was not an inevitable outcome after all. Similar studies of other lexical fields find different principles structuring different domains. For example, Shepard and Cooper (1992) found that hue was the most important property structuring the domain of color. Storm (1980) discovered that size and habitat (land vs. water) were the underlying dimensions of an animal lexicon. Fillenbaum and Rapoport (1971) found person and number to be the most important properties organizing the lexical field of pronouns. And so forth. Thus, the parallelism between the 
odor lexicon and emotion lexicon is notable. This could reflect the well-documented close relationship between odors and emotions. Verbal descriptions of olfactory sensations in Western languages often involve affective terminology (Dubois, 2000). And outside of language olfaction and emotion are closely associated too (e.g., Herz \& Engen, 1996). Here, for the first time, we show that the semantic field of a dedicated olfactory lexicon can also parallel the structure of emotion lexicons.

Does the fact that we found a 2-dimensional solution for the Maniq smell lexicon mean that the theory of unidimensional odor representations or those involving more than 2 dimensions are wrong? And could our data support alternative theories of odor perception? Assuming that language structure mirrors perceptual structure, we may treat this finding as evidence against the unidimensional and highdimensional models of odor perception. A different interpretation of our results would be to assume that olfactory language is not a faithful reflection of olfactory psychophysics, but that other considerations (perhaps culture-specific) play a role in forming olfactory categories in language. This then raises the question of what exactly the relationship is between odor perception and odor language. Are these completely independent systems? Does smell language use the same representations as the perceptual system? And the other way round, can smell language influence smell perception? We leave these questions for future studies to address.

\section{Conclusion}

The cultural and linguistic elaboration of smell among the Maniq constitutes compelling evidence against the universal paucity of olfactory terms, the "weak link" between smell and language, and the general insignificance of olfaction for humans. This evidence is even stronger in light of the fact that Maniq is not an isolated case of this kind of elaboration. There are a number of similar smell-oriented cultures around the world (e.g. Burenhult \& Majid, 2011; Classen et al., 1994; Majid \& Burenhult, 2014). These cultures have so far been largely overlooked. By embracing cultural and linguistic diversity, we can make significant advances in understanding human olfaction that otherwise would not emerge. These results resonate with recent evidence that the human sense of smell is more acute than previously believed (Laska, Seibt, \& Weber, 2000; Shepherd, 2004), and that smell is not a vestigial sense, but serves a variety of functions (Stevenson, 2010). The data presented here urge us to modify our perspective on yet another dogma of olfaction. Human languages can encode odors.

\section{Acknowledgements}

We are grateful to the Maniq community of Manang (Satun), the National Research Council of Thailand, Jaroon Thotsagool, Theraphan Luangthongkum and Suwilai Premsrirat. We thank Niclas Burenhult, Nicole Kruspe, Katesarin Maneenoon, Stephen Levinson, Rebecca Defina, Saskia van Putten and Rósa Gísladóttir. This work was supported by Max Planck Gesellschaft and the NWO grant "Human olfaction at the intersection of language, culture and biology" awarded to the second author.

\section{References}

Ayabe-Kanamura, S., Schicker, I., Laska, M., Hudson, R., Distel, H. Kobayakawa, T., et al (1998). Differences in perception of everyday odors: A Japanese-German cross-cultural study. Chemical Senses, 23(1), 31-38. http://dx.doi.org/10.1093/chemse/23.1.31.

Beek, W. E. van (1992). The dirty smith: Smell as a social frontier among the Kapsiki/Higi of north Cameroon and north-eastern Nigeria. Africa, 38-58.

Beer, B. (2007). Smell, person, space and memory. In J. Wassmann \& K. L. Stockhaus (Eds.). Experiencing new worlds (Vol. 1, pp. 187-200). New York: Berghahn Books.

Beller, S., Bender, A., \& Medin, D. L. (2012). Should anthropology be part of cognitive science? Topics in Cognitive Science, 4(3), 342-353. http://dx. doi.org/10.1111/j.1756-8765.2012.01196.x.

Bender, A., Hutchins, E., \& Medin, D. (2010). Anthropology in cognitive science. Topics in Cognitive Science, 2(3), 374-385. http://dx.doi.org/ 10.1111/j.1756-8765.2010.01082.x.

Benjamin, G. (1985). In the long term: Three themes in Malayan cultural ecology. In K. L. Hutterer, A. T. Rambo, \& G. W. Lovelace (Eds.), Cultural values and human ecology in Southeast Asia (pp. 219-278). Ann Arbor, MI: University of Michigan, Center for South and Southeast Asian Studies.

Berlin, B. (1992). Ethnobiological classification: Principles of categorization of plants and animals in traditional societies. Princeton, NJ: Princeton University Press.

Bishop, N., \& Peterson, M. (2003). Northern Aslian language survey: Trang, Satul and Phatthalung provinces, Thailand. Bangkok: TU-SIL-LRDP Thammasat University.

Boster, J. S., \& Johnson, J. C. (1989). Form or function: A comparison of expert and novice judgments of similarity among fish. American Anthropologist, 91(4), 866-889.

Burenhult, N., \& Majid, A. (2011). Olfaction in Aslian ideology and language. The Senses and Society, 6(1), 19-29.

Burton, R. (1976). The language of smell. London: Routledge \& Kegan Paul. Burton, M. L., \& Nerlove, S. B. (1976). Balanced designs for triads tests: Two examples from English. Social Science Research, 5(3), 247-267.

Caballero, R. (2007). Manner-of-motion verbs in wine description. Journal of Pragmatics, 39(12), 2095-2114. http://dx.doi.org/10.1016/j.pragma. 2007.07.005.

Cain, W. S. (1979). To know with the nose: Keys to odor identification. Science, 203(4379), 467-470. http://dx.doi.org/10.1126/science. 760202 .

Cain, W. S., de Wijk, R., Lulejian, C., Schiet, F., \& See, L. C. (1998). Odor identification: Perceptual and semantic dimensions. Chemical Senses, 23(3), 309-326.

Casson, R. W. (1997). Color shift: Evolution of English color terms from brightness to hue. In C. L. Hardin \& L. Maffi (Eds.), Color categories in thought and language (pp. 320-346). Cambridge University Press.

Cattell, R. B. (1966). The scree test for the number of factors. Multivariate Behavioral Research, 1(2), 245-276.

Chrea, C., Valentin, D., Sulmont-Rossé, C., Ly Mai, H., Hoang Nguyen, D., \& Abdi, H. (2004). Culture and odor categorization: Agreement between cultures depends upon the odors. Food Quality and Preference, 15(7-8), 669-679.

Classen, C., Howes, D., \& Synnott, A. (1994). Aroma: The cultural history of smell. London: Routledge.

Condillac, E. B. de. (1930). Condillac's treatise on the sensations (M. G. S. Carr, Trans.). London: The Favil Press. (Original work published 1754).

Dallos, C. (2011). From equality to inequality: Social change among newly sedentary Lanoh hunter-gatherer traders of Peninsular Malaysia. Toronto: University of Toronto Press.

Darwin, C. (1874). The descent of man, and selection in relation to sex (2nd ed.). London: John Murray.

Distel, H., \& Hudson, R. (2001). Judgement of odor intensity is influenced by subjects' knowledge of the odor source. Chemical Senses, 26(3), $247-251$.

Doty, R. L. (1975). An examination of relationships between the pleasantness, intensity, and concentration of 10 odorous stimuli. Perception \& Psychophysics, 17(5), 492-496.

Drake, M. A., \& Civille, G. V. (2003). Flavor lexicons. Comprehensive Reviews in Food Science and Food Safety, 2(1), 33-40. http://dx.doi.org/10.1111/ j.1541-4337.2003.tb00013.x.

Dubois, D. (2000). Categories as acts of meaning: The case of categories in olfaction and audition. Cognitive Science Quarterly, 1(1), 35-68. 
Endicott, K. M. (1979). Batek Negrito religion: The world-view and rituals of a hunting and gathering people of Peninsular Malaysia. Oxford: Clarendon Press.

Fillenbaum, S., \& Rapoport, A. (1971). Structures in the subjective lexicon. New York: Academic Press.

Fillenbaum, S., \& Rapoport, A. (1974). Verbs of judging, judged: A case study. Journal of Verbal Learning and Verbal Behavior, 13(1), 54-62. http://dx.doi.org/10.1016/S0022-5371(74)80030-7.

Gardner, H. (1993). Frames of mind: The theory of multiple intelligences. New York, NY: Basic Books.

Gluck, M. (2003). Wine language: Useful idiom or idiot-speak? In J. Aitchison \& D. M. Lewis (Eds.), New media language (pp. 108-115). London: Routledge.

Grinker, R. R. (1934). Neurology. Springfield, IL: C.C. Thomas.

Haddad, R., Weiss, T., Khan, R., Nadler, B., Mandairon, N., Bensafi, M., et al (2010). Global features of neural activity in the olfactory system form a parallel code that predicts olfactory behavior and perception. The Journal of Neuroscience, 30(27), 9017-9026. http://dx.doi.org/10.1523/ JNEUROSCI.0398-10.2010.

Hayton, J. C., Allen, D. G., \& Scarpello, V. (2004). Factor retention decisions in exploratory factor analysis: A tutorial on parallel analysis. Organizational Research Methods, 7(2), 191-205.

Henning, H. (1916). Der Geruch. Leipzig: J.A. Barth.

Henrich, J., Heine, S. J., \& Norenzayan, A. (2010). The weirdest people in the world. Behavioral and Brain Sciences, 33(2-3), 61-83.

Herz, R. S., \& Engen, T. (1996). Odor memory: Review and analysis. Psychonomic Bulletin \& Review, 3(3), 300-313. http://dx.doi.org/ $10.3758 /$ BF03210754.

Hombert, J.-M. (1992). Terminologie des odeurs dans quelques langues du Gabon. Pholia.

Jönsson, F. U., \& Olsson, M. J. (2012). Knowing what we smell. In G. Zucco R. Herz, \& B. Schaal (Eds.), Olfactory cognition: From perception and memory to environmental odours and neuroscience (pp. 115-135). Amsterdam, Philadelphia: John Benjamins Publishing Company.

Kaeppler, K., \& Mueller, F. (2013). Odor classification: A review of factors influencing perception-based odor arrangements. Chemical Senses, 38 (3), 189-209. http://dx.doi.org/10.1093/chemse/bjs141.

Kaiser, H. F. (1960). The application of electronic computers to factor analysis. Educational and Psychological Measurement, 20, 141-151. http://dx.doi.org/10.1177/001316446002000116.

Kant, I. (2006). Anthropology from a pragmatic point of view (R. B. Louden, Trans.). Cambridge: Cambridge University Press. (Original work published 1798)

Khan, R. M., Luk, C.-H., Flinker, A., Aggarwal, A., Lapid, H., Haddad, R., et al (2007). Predicting odor pleasantness from odorant structure: Pleasantness as a reflection of the physical world. Journal of Neuroscience, 27(37), 10015-10023. http://dx.doi.org/10.1523/ JNEUROSCI.1158-07.2007.

Koulakov, A. A., Kolterman, B. E., Enikolopov, A. G., \& Rinberg, D. (2011). In search of the structure of human olfactory space. Frontiers in Systems Neuroscience, 5, 1-8. http://dx.doi.org/10.3389/fnsys.2011.00065.

Kruskal, J. B. (1964). Multidimensional scaling by optimizing goodness of fit to a nonmetric hypothesis. Psychometrika, 29(1), 1-27.

Kruskal, J. B., \& Wish, M. (1978). Multidimensional scaling. Beverly Hills, CA: Sage.

Laska, M., Seibt, A., \& Weber, A. (2000). "Microsmatic" primates revisited: Olfactory sensitivity in the squirrel monkey. Chemical Senses, 25(1), 47-53. http://dx.doi.org/10.1093/chemse/25.1.47.

Lawless, H. T., \& Cain, W. S. (1975). Recognition memory for odors. Chemical Senses, 1(3), 331-337. http://dx.doi.org/10.1093/chemse/ 1.3.331.

Lawless, H., \& Engen, T. (1977). Associations to odors: Interference, mnemonics, and verbal labeling. Journal of Experimental Psychology: Human Learning and Memory, 3(1), 52.

Lee, A. P. (2010). Reduplication and odor in four Formosan languages. Language and Linguistics, 11(1), 99-126.

Lehrer, A. (1983). Wine and conversation. Oxford: Oxford University Press.

Lehrer, A. (2009). Wine and conversation (2nd ed.). Oxford, New York: Oxford University Press.

Levinson, S. C. (2012). The original sin of cognitive science. Topics in Cognitive Science, 4(3), 396-403. http://dx.doi.org/10.1111/j.17568765.2012.01195.x.

Levinson, S. C., \& Majid, A. (in press). Differential ineffability and the senses. Mind \& Language.

Lyons, J. (1977). Semantics. Cambridge, New York: Cambridge University Press.

Madany Mamlouk, A., Chee-Ruiter, C., Hofmann, U. G., \& Bower, J. M. (2003). Quantifying olfactory perception: Mapping olfactory perception space by using multidimensional scaling and selforganizing maps. Neurocomputing, 52, 591-597.

Majid, A. (in press). Comparing lexicons cross-linguistically. In J. R. Taylor (Ed.), The Oxford handbook of the word. Oxford University Press.

Majid, A., \& Burenhult, N. (2014). Odors are expressible in language, as long as you speak the right language. Cognition, 130(2), 266-270. http://dx. doi.org/10.1016/j.cognition.2013.11.004.

Majid, A., \& van Staden, M. (2013). Ain't got no hands, ain't got no feet: Variation of body part categories in three unrelated languages. Manuscript submitted for publication.

Majid, A. (2010). Words for parts of the body. In B. C. Malt \& P. M. Wolff (Eds.), Words and the mind: How words capture human experience (pp. 58-71). New York: Oxford University Press.

Malt, B. C., Ameel, E., Imai, M., Gennari, S., Saji, N., \& Majid, A. (in press). Human locomotion in languages: Constraints on moving and meaning. Journal of Memory and Language.

Malt, B. C., Gennari, S., Imai, M., Ameel, E., Tsuda, N., \& Majid, A. (2008). Talking about walking: Biomechanics and the language of locomotion. Psychological Science, 19(3), 232-240. http://dx.doi.org/10.1111/ j.1467-9280.2008.02074.x.

Maneenoon, K. (2001). Ethnobotany of Sakai tribe in Trang, Phatthalung and Yala provinces. Master's thesis. Prince of Songkla University, Hat Yai.

Maneenoon, K., Sirirugsa, P., \& Sridith, K. (2008). Ethnobotany of Dioscorea L. (Dioscoreaceae), a major food plant of the Sakai tribe at Banthad Range, Peninsular Thailand. Ethnobotany Research \& Applications, 6, 385-394.

Martínez-Mayorga, K., Peppard, T. L., Yongye, A. B., Santos, R., Giulianotti, M., \& Medina-Franco, J. L. (2011). Characterization of a comprehensive flavor database. Journal of Chemometrics, 25(10), 550-560. http://dx. doi.org/10.1002/cem.1399.

Medin, D. L., \& Atran, S. (1999). Folkbiology. Cambridge, Mass.: MIT Press.

O'Connor, B. P. (2000). SPSS and SAS programs for determining the number of components using parallel analysis and Velicer's MAP test. Behavior Research Methods, Instruments, E Computers, 32(3), 396-402.

Phaiboon, D. (2006). Glossary of Aslian languages: The northern Aslian languages of southern Thailand. Mon-Khmer Studies, 36, 207-224.

Pinker, S. (1997). How the mind works. New York: Norton.

Quandt, R. E. (2007). On wine bullshit: Some new software? Journal of Wine Economics, 2, 129-135.

Romney, A. K., Weller, S. C., \& Batchelder, W. H. (1986). Culture as consensus: A theory of culture and informant accuracy. American Anthropologist, 88(2), 313-338. http://dx.doi.org/10.2307/677564.

Rouby, C., \& Bensafi, M. (2002). Is there a hedonic dimension to odors? In C. Rouby, B. Schaal, D. Dubois, R. Gervais, \& A. Holley (Eds.), Olfaction, taste, and cognition (pp. 140-159). New York: Cambridge University Press.

Russell, J. A. (1980). A circumplex model of affect. Journal of Personality and Social Psychology, 39(6), 1161.

Russell, J. A., Lewicka, M., \& Niit, T. (1989). A cross-cultural study of a circumplex model of affect. Journal of Personality and Social Psychology, 57(5), 848-856. http://dx.doi.org/10.1037/0022-3514.57.5.848.

Ruts, W., De Deyne, S., Ameel, E., Vanpaemel, W., Verbeemen, T., \& Storms, G. (2004). Dutch norm data for 13 semantic categories and 338 exemplars. Behavior Research Methods, 36(3), 506-515.

Saussure, F. de (1967). Cours de linguistique générale. Wiesbaden: Harrassowitz.

Shepard, G. H., Jr. (1999). Pharmacognosy and the senses in two Amazonian societies. PhD dissertation. University of California, Berkeley.

Shepard, R. N., \& Cooper, L. A. (1992). Representation of colors in the blind, color-blind, and normally sighted. Psychological Science, 3(2), 97-104.

Shepherd, G. M. (2004). The human sense of smell: Are we better than we think? PLoS Biology, 2(5), e146. http://dx.doi.org/10.1371/journal. pbio.0020146.

Sperber, D. (1975). Rethinking symbolism (A. L. Morton, Trans.). Cambridge: Cambridge University Press. (Original work published 1974).

Stanley-Jones, D. (1957). Posture and the rhinencephalon: A new interpretation. The Journal of Nervous and Mental Disease, 125(4), 591-598.

Stevenson, R. J. (2010). An initial evaluation of the functions of human olfaction. Chemical Senses, 35(1), 3-20. http://dx.doi.org/10.1093/ chemse/bjp083.

Storchmann, K. (2011). Wine economics: Emergence, developments, topics. Agrekon, 50(3), 1-28. http://dx.doi.org/10.1080/03031853.2011.617856.

Storm, C. (1980). The semantic structure of animal terms: A developmental study. International Journal of Behavioral Development, 3(4), 381-407. http://dx doi.org/10.1177/016502548000300403.

Sturrock, K., \& Rocha, J. (2000). A multidimensional scaling stress evaluation table. Field Methods, 12(1), 49-60. http://dx.doi.org/ $10.1177 / 1525822$ X0001200104. 
Tufvesson, S. (2011). Analogy-making in the Semai sensory world. The Senses and Society, 6(1), 86-95. http://dx.doi.org/10.2752/ $174589311 X 12893982233876$.

Weil, R. L. (2007). Debunking critics' wine words: Can amateurs distinguish the smell of asphalt from the taste of cherries? Journal of Wine Economics, 2(02), 136-144. http://dx.doi.org/10.1017/ S1931436100000390.

Weller, S. C., \& Romney, A. K. (1988). Systematic data collection. Newbury Park, CA: Sage Publications.

Wilson, D. A., \& Stevenson, R. J. (2006). Learning to smell: Olfactory perception from neurobiology to behavior. Baltimore: Johns Hopkins University Press.
Wnuk, E. (2013). Semantic specificity in verbs of perception in the context of ethnobiology in Maniq. PhD dissertation. Manuscript in preparation.

Wnuk, E., \& Burenhult, N. (2013). The sound system of Maniq, an Aslian outlier of Isthmian Thailand. Manuscript submitted for publication.

Yeshurun, Y., \& Sobel, N. (2010). An odor is not worth a thousand words: From multidimensional odors to unidimensional odor objects. Annual Review of Psychology, 61, 219-241.

Zarzo, M. (2008). Psychologic dimensions in the perception of everyday odors: Pleasantness and edibility. Journal of Sensory Studies, 23(3), $354-376$. 